\title{
Revisión
}

La cooperación internacional en el

ciberperiodismo ecuatoriano.

Los casos de

Plan

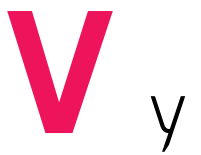

Periodismo de

Investigación

\section{Saudia Levoyer}

saudia.levoyer auasb.edu.ec

Recibido: 28 de julio de 2020 | Aceptado: 11 de agosto de 2020

D0I: https://doi.org/10.18272/pd.v4i1.1905

Referencia de este artículo:

Levoyer, S. (2020). La cooperación internacional en el desarrollo del ciberperiodismo de Ecuador. Los casos de los portales Plan V y Periodismo de Investigación. \#PerDebate, volumen 4 (pp. 182-197). Quito: USFQ Press.

Saudia Levoyer es periodista, docente universitaria y de postgrado. Doctora en Comunicación e Información Contermporánea, Máster en Estudios Latinoamericanos mención Estudios de la Cultura, Licenciada en Comunicación. Profesora de la Universidad Andina Simón Bolívar, sede Ecuador. 


\title{
Resumen
}

La expansión de los cibermedios ecuatorianos contó con el apoyo de la cooperación internacional, interesada en el fortalecimiento del sistema democrático y la libertad de expresión. Las ONG internacionales, con apoyo de las que se encuentran en Ecuador, invirtieron en capacitación y capitales semilla. Este proceso arrancó cuando la relación entre el gobierno de Rafael Correa (2007-2017) y los medios de comunicación de información se tensó y los periodistas migraron a la web, para continuar con su trabajo.

Plan V y Periodismo de Investigación son dos de los proyectos que recibieron fondos para desarrollarse. Esta forma de financiamiento puede considerarse como una variante del modelo de negocio patrocinado.

Esta investigación, que es de tipo descriptivo, busca determinar cómo se establece la ayuda de la cooperación internacional a los cibermedios, para lo cual se realizaron entrevistas a profundidad a los voceros de las ONG locales, como Fundamedios y FOPE; una internacional, como Freedom House; y a los representantes de los cibermedios Plan V y Periodismo de Investigación. Este trabajo forma parte de una investigación financiada por la Universidad Andina Simón Bolívar, sede Ecuador.

\section{Palabras clave}

cibermedios, modelos de negocio, financiamiento, cooperación internacional

\section{International cooperation in Ecuadorian cyberjournalism. The cases of Plan V and Pariodismo de Investigación}

\begin{abstract}
The expansion of Ecuadorian online media had the support of international cooperation, interested in strengthening the democratic system and freedom of speech. Internacional NGOs, with the support of those in Ecuador, invested in training and seed capital. This process started when the relationship between the Rafael Correa government (2007-2017) and the media became strained and journalists migrated to the web to continue their work.

Plan V and Periodismo de Investigación are two of the projects that received development funding. This form of financing is a variant of the sponsored business model. This research, which is descriptive in nature, seeks to determine how international cooperation aid to online media is established, for which in-depth interviews were conducted with the spokesperson of local and international NGOs, such as Fundamedios and Foro de Periodismo del Ecuador; an international one, such as Freedom House; and representatives of Plan $V$ and Periodismo de Investigación online media. This work is part of an investigation financed by Univerisidad Andina Simón Bolívar, Ecuador.
\end{abstract}

\section{Keywords}

cyberjournalism, business models, financing, international cooperation 


\section{Introducción}

El aparecimiento y posterior desarrollo de los portales informativos o cibermedios en Ecuador han contado con el apoyo de la cooperación internacional y sus agencias de desarrollo. El origen de este tipo de financiamiento encuentra una de sus explicaciones en la tensa y compleja relación que surgió y se mantuvo entre la prensa y el presidente Rafael Correa (2007-2017), que llevó a que muchos periodistas encontraran en la web el espacio propicio para desarrollar y continuar con su trabajo.

Las organizaciones no gubernamentales (ONG) nacionales, como Fundamedios y el Foro de Periodistas del Ecuador (FOPE), que trabajan en la promoción de las libertades de expresión y de prensa, se convirtieron en el centro de enlace entre las ONG internacionales y los cibermedios, que han solicitado apoyo especialmente en capacitación y financiamiento, para continuar con su trabajo periodístico.

Esta nueva forma de obtener dinero, en un primer momento, no influyó en la agenda de los cibermedios, ya que tanto estos como las ONG estaban alineadas en la lucha por el fortalecimiento democrático. Sin embargo, conforme ha cambiado la situación política del Ecuador, esos temas ya no atraen la atención de los aportantes, lo que ha llevado a que los portales también se interesen en los temas que están en la lista de prioridades de esas organizaciones, como son migración, violencia contra la mujer, entre otros.

El presente artículo es un estudio de caso de los portales Plan $V$ y Periodismo de Investigación, que muestra y describe cómo funciona este financiamiento y cómo ha continuado hasta el momento, aunque en menores montos. También establece cómo han girado su agenda temática o setting para continuar participando en los concursos que entregan dinero para temas vinculados a los derechos humanos.

Vale recalcar que los resultados que aquí se muestran son parte de una investigación más amplia que se desarrolló en el Comité de Investigaciones de la Universidad Andina Simón Bolívar, sede Ecuador.

\section{Marco teórico}

\subsection{Cibermedios y modelos de negocios}

El ciberperiodismo es una especialidad del periodismo, que utiliza el ciberespacio para investigar y producir contenidos de tipo periodístico, así como difundirlos, a través de diferentes plataformas (Salaverría, 2005). La existencia de esta variedad de plataformas está atada a que el negocio de la información va de la mano con la tecnología, que no solo ha modificado la producción y distri- 
bución de información, sino también el consumo y la relación con los usuarios, por la personalización e individualización al momento de acceder al contenido. Esto, a su vez, constituye una estrategia clave para la captación de nuevos usuarios o la fidelización de los existentes (López y Pereira, 2008).

Una de las mayores dificultades que han afrontado los cibermedios es cómo lograr un financiamiento que les permita subsistir, es decir, tener un modelo exitoso de negocio. Picard (2011) plantea a la publicidad, el pago por contenido, el patrocinio y la demanda como alternativas para conseguir dinero. En esto también concuerda Campos Freire (2015) y agrega que, así, también se puede generar un equilibrio en las ganancias entre los financistas y los trabajadores del medio digital.

Hay otras propuestas como la de Pavlik (2008), quien sugiere una mezcla de modelos - como una forma alternativa de obtención de fondos-, pero sustentada en las audiencias, en la forma de producción de los contenidos y en la plataforma de distribución.

Portilla Manjón, Vara-Miguel y Díaz-Espina (2016) comparten la propuesta de Pavlik, ya que insisten en que los niveles de innovación vienen de la mano del modelo económico y que, en consecuencia, esto lleva al desarrollo del periodismo en la red y la sostenibilidad financiera del emprendimiento o de la empresa. El pago por conocer más un contenido es uno de los mecanismos clave, aunque ellos agregaron el crowdfunding, los muros de pagos y la membresía.

Sábada (2016) plantea la idea del trabajo en red que, por un lado, lleva a que un medio pueda contar con una plana de redactores (aunque también señala que se puede provocar la precarización del trabajo) y, por otro lado, a que el grupo de usuarios, la oferta de datos y la estructura digital impulse a que el cibermedio se reinvente y busque más asociaciones estratégicas.

El estudio de Punín, Martínez y Rencoret (2013), respecto a la situación de los medios digitales en el Ecuador, señala que el modelo que prima es el publicitario, en el cual los cibermedios ubican la publicidad con base en una pauta anual, que es mayoritariamente comprada por el Estado. Esto a pesar de que, como señalan Escandón-Montenegro y Levoyer (2019), no son considerados al mismo nivel que los medios de comunicación tradicionales y no son vistos como potenciales difusores de la publicidad estatal.

Pero también hay los cibermedios patrocinados, entre los que se puede ubicar a los que reciben cooperación internacional, y que enfocan su agenda setting alrededor de los Objetivos de Desarrollo Sostenible (ODS), los derechos humanos, el fortalecimiento a la democracia y las libertades. 


\subsection{Prensa, cooperación internacional y gobiernos autoritarios}

El neopopulismo que afronta América Latina desde inicios del siglo XXI comparte varias similitudes. Una de ella es la politización de la información y el papel de los medios de comunicación, que plantean Omar Rincón y Carlos de la Torre.

El primero señala que los gobiernos tienen una fascinación por la lógica mediática y que los medios no quieren perder el dominio de la opinión pública (Rincón, 2010). El segundo, que los medios juegan un papel político y que, por ejemplo, en el caso ecuatoriano esto se ha evidenciado en las caídas de los presidentes Abdalá Bucaram (1997), Jamil Mahuad (2000) y Lucio Gutiérrez (2005) (De la Torre, 2015).

Los gobiernos de Rafael Correa (Ecuador), Hugo Chávez (Venezuela), Evo Morales (Bolivia), Néstor Kirchner y Cristina Fernández de Kirchner (Argentina) mantuvieron una relación con los periodistas caracterizada por el ataque a su credibilidad y la de los medios; la inclusión de los insultos y las descalificaciones a los periodistas en el discurso político; la creación de sus propios medios de comunicación; la aprobación de legislación que generó reacciones contrarias de la prensa (Amado, 2015; Álvarez y Chumaceiro, 2012).

En el caso ecuatoriano incluso se llegó a la suspensión de la publicidad estatal y a la prohibición de brindar declaraciones públicas de los funcionarios de Estado a los medios de comunicación considerados como opositores por el gobierno de Correa (Levoyer, 2019). Además, de procesos judiciales que terminaron en sentencias millonarias en contra de periodistas, medios de comunicación y sus dueños (caso El Universo, que ordenaba el pago de USD 40000 000 al presidente Correa por dañar su honra), así como penas de privación de la libertad (los casos están recogidos en los informes de las Relatorías de la Libertad de Expresión de 2012, 2015 y 2017).

En lo que respecta a la cooperación internacional, Ecuador dio un giro en su política exterior y, en consecuencia, en los lineamientos brindados al trabajo de las organizaciones internacionales y cooperantes que desarrollaban actividades o apoyo a las ONG nacionales. Cómo debía entenderse la política exterior ecuatoriana se encuentra en Rodríguez Gelfenstein (2013) y en el Plan Nacional de Desarrollo (2007-2010), y las evidencias del cumplimiento de esa nueva concepción en Ayllón y Dolcetti (2014).

Rodríguez Gelfenstein (2013) señala que se apostó por el fortalecimiento de un orden internacional multipolar, lo cual implicaría un proceso de robustecimiento de alianzas prioritarias, especialmente a través de la integración latinoamericana y Sur-Sur. En el Plan Nacional de Desarrollo (2007-2010) se ratifica que el objetivo es garantizar la soberanía nacional, la paz y auspiciar la integración latinoamericana, entre otros. Y las evidencias de las que hablan Ayllón y Dolce- 
tti (2014) están, por ejemplo, en la salida de los soldados estadounidenses de la Base Aérea de Manta; el rechazo del Gobierno a las iniciativas de libre comercio impulsadas por Estados Unidos; y el ingreso de Ecuador a la Alianza Bolivariana para los Pueblos de Nuestra América (ALBA).

A nivel de las ONG, que juegan el papel de intermediarias frente a la cooperación internacional, la visión gubernamental se plasmó en la Agencia Ecuatoriana de Cooperación Internacional (AGECI), que debía ejecutar "las estrategias generales de cooperación internacional, las políticas y reglamentos de gestión, el desarrollo y aplicación de instrumentos de gestión del Sistema Ecuatoriano de Cooperación Internacional" (Registro Oficial Nro. 113, 2007). Estas, como se señaló anteriormente, priorizaban la cooperación Sur-Sur, con lo cual aportantes del norte, por ejemplo, Estados Unidos, veían cómo su presencia sufría cada vez más limitaciones.

La AGECl, tres años más tarde, mediante Decreto Ejecutivo Nro. 429, de 15 de julio 2010, se convirtió en la Secretaría Técnica de Cooperación Internacional (SETECI), que tuvo a su cargo cumplir con los polémicos decretos ejecutivos 16 y 739, del 4 de junio de 2013 y del 3 de agosto de 2015, respectivamente. En ellos se planteaba la disolución de dos organizaciones de la sociedad civil (Fundación Pachamama y la Unión Nacional de Educadores) por no cumplir con los fines para los que fueron constituidas, dedicarse a actividades político partidistas y atentar contra la seguridad del Estado, como consta en las notificaciones hechas a esos organismos y denunciados en los medios de comunicación de la fecha.

Luego de ejecutar lo ahí establecido, se advirtió sobre el inicio de procesos similares a la Confederación de Nacionalidades Indígenas del Ecuador (CONAIE) y a la Fundación Andina para la Observación y Estudio de Medios (Fundamedios). La primera es la organización indígena más importante del Ecuador y la segunda, desde 2007, trabajaba por la libertad de expresión y de prensa, y era referente de las Relatorías de la Libertad de Prensa de la de la Organización de las Naciones Unidas (ONU) y de la Organización de Estados Americanos (OEA), para conocer lo que ocurría en el Ecuador en estas áreas, además de ser el enlace con los nacientes cibermedios ecuatorianos.

\section{Metodología}

Al tratarse de un trabajo descriptivo, se escogió la técnica cualitativa de la entrevista, con los informantes calificados de las ONG, tanto nacionales como internacionales y los directivos de los cibermedios.

Mendieta Vicuña y Esparcia Pérez (2018), quienes citan a Valles (2017), Kvale (2011) y Arocena (2001), señalan que la entrevista es útil para comprender la 
manera por la cual el entrevistado percibe la realidad, a fin de generar conocimiento empírico.

Los primeros diálogos fueron entre octubre y noviembre de 2019, con los representantes de Fundamedios, Foro de Periodistas del Ecuador (FOPE) y el vocero de Freedom House. Estas organizaciones apoyaron el trabajo de la prensa desde 2007. Hay que aclarar que también se estableció contacto con Panamerican Development Foundation (PADF), pero no aceptaron participar en el estudio, aduciendo falta de tiempo para atender a la entrevista.

Con los voceros de las ONG nacionales se dialogó sobre su relación con los cooperantes y, a su vez, con los cibermedios. Al delegado de Freedom House, sobre por qué se apostó por esta ayuda y qué tan extendida fue para la prensa del Ecuador.

Los representantes de los cibermedios con quienes se conversó en diciembre de 2019 fueron los directivos de los portales Plan V, Juan Carlos Calderón, y Periodismo de Investigación, Christian Zurita. Los dos portales trabajan en investigación de corrupción y seguridad. Ambos entrevistados cuentan con una amplia experiencia en la prensa y se han desempeñado como director de medios, el primero, y como redactor especial, el segundo. Las temáticas abordadas fueron: surgimiento del cibermedio y el contexto político en el que ocurrió; la forma de financiamiento; las organizaciones internacionales que les brindaron apoyo; los requisitos que debieron cumplir para aplicar a la consecución de fondos; el tipo de propuestas que enviaron; y la relación entre el financiamiento y la injerencia en la política editorial.

\section{Resultados}

Con base en las entrevistas realizadas, se puede decir que la web se convirtió en el espacio alterno para los periodistas que debieron dejar los medios de comunicación masivos, ante la persecución a la prensa, a los dirigentes sociales y a las organizaciones de la sociedad civil, por parte del gobierno de Rafael Correa. A ello se suma el recelo que hubo en algunos de los medios de comunicación de mantener líneas periodísticas que pudieran parecer de confrontación, lo que también llevó a que los periodistas dejen esos medios. En la década 2007-2017, las leyes para controlar y sancionar el trabajo periodístico, junto con los procesos judiciales, la censura, la autocensura y el acoso político, se convirtieron en una práctica gubernamental. Esta política autoritaria se fue extendiendo poco a poco hasta alcanzar a los dirigentes políticos y sociales, así como a las ONG. 
Los periodistas, que salieron por su propia voluntad o por presiones políticas de los medios de comunicación tradicionales, arribaron al mundo digital, para continuar con su trabajo. Esta migración produjo un ecosistema mediático en el cual se dio cabida a las distintas iniciativas de periodismo independiente dado que las regulaciones legales aprobadas no tocaban a los cibermedios. Los nacientes medios digitales, sin embargo, no tenían una idea clara de cómo conseguir dinero y, en consecuencia, cómo trazar un proyecto de negocios, de crecimiento, de promoción y de mercadeo.

Fundamedios, que nació en 2007, entonces comenzó a jugar un papel distinto al que venía desempeñando: amplió su campo de acción de monitorear las agresiones en contra de los periodistas a apuntalar la creación de los cibermedios, a través de la capacitación y la consecución de fondos extranjeros, vía participación en concursos de proyectos. A la tarea de formación se le unió más tarde (hacia 2012) el Foro de Periodistas del Ecuador (FOPE).

Fundamedios, hasta 2019, manejó USD 2000000 y FOPE USD 45 000, que provinieron de los Departamento de Relaciones Internacionales y de Comunicación de la Embajada de Estados Unidos, del Departamento de Comunicación de la Embajada de Canadá, de Freedom House, de Naciones Unidas, de Open Society Institute (hoy Open Society Foundation), de la Corporación Andina de Fomento (CAF), de la Fundación Avina, de National Endowment for Democracy (NED) y de la Unión Europea.

Los cibermedios que han recibido capital semilla son los portales Plan V, GK y 4Pelagatos. Pero también hay iniciativas, especialmente a partir de finales de 2016 e inicios de 2017, de entrega de becas, por un máximo de USD 2000, para que los periodistas impulsen su labor de investigación.

En cuanto a la agenda temática de los medios y la incidencia de la cooperación internacional, se pueden establecer dos momentos muy claros. El primero fue cuando el Ecuador entró en el radar de los organismos internacionales, gracias al trabajo de Fundamedios de dotar de información a las Relatorías de la Libertad de Expresión de la ONU y la OEA. Sus denuncias sobre las violaciones a las libertades de expresión y de prensa permitieron que las ONG se preocuparan de la situación del Ecuador y se interesaran por promocionar esas libertades y el fortalecimiento del sistema democrático. Por ello, generaron fondos para apoyar a los nacientes cibermedios ecuatorianos, que estaban concentrados en publicar temáticas que no siempre se abordaban en los medios masivos tradicionales de radio, prensa y televisión. En esta etapa el objetivo era que los periodistas continúen con su trabajo y la agenda que abordaban era decisión de ellos. Los temas en los que se concentraron fue la lucha contra la corrupción, las garantías políticas, la libertad de expresión y los derechos humanos. 


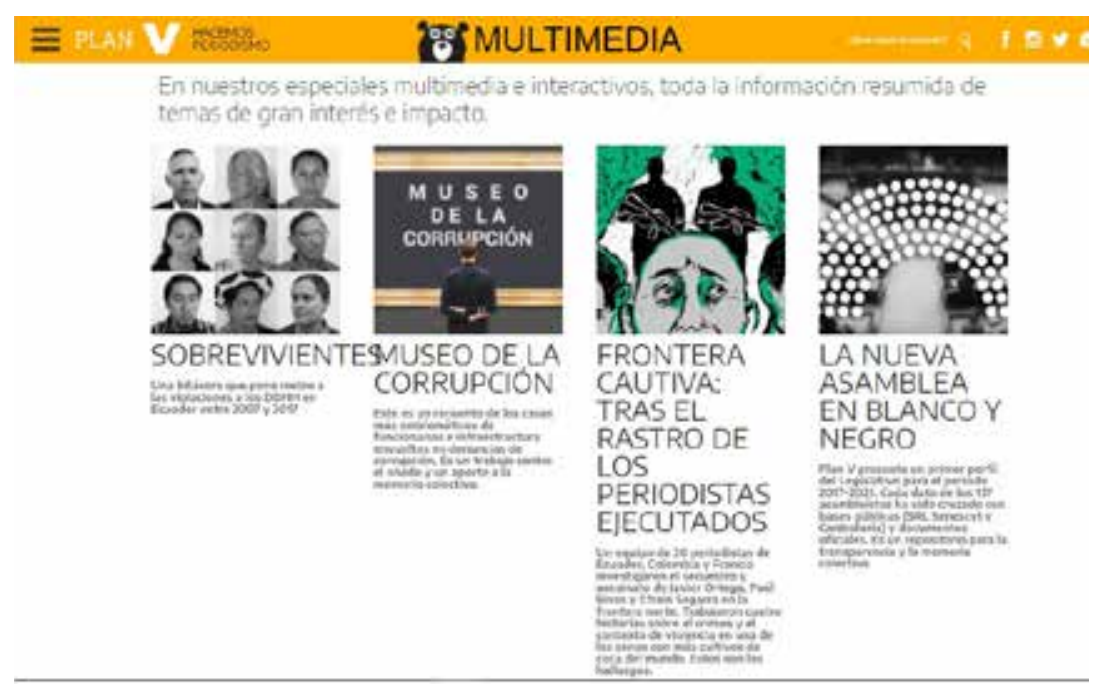

Estos son algunos de los reportajes especiales de Plan V, que tratan sobre corrupción, política y derechos humanos. Este cibermedio plantea temáticas amplias alrededor de tópicos que pueden tener financiamiento de cooperación.

Jaime Veintimilla, representante en Ecuador de Freedom House, admite que las ONG tuvieron un accionar reactivo y no preventivo, ya que no se habían preocupado antes de la libertad de prensa y expresión, sino hasta que se produjo la violación de estos derechos, lo que llevó también a que consiguieran dinero más fácilmente. Esto explica los apoyos puntuales y no sistemáticos que se han dado a estos temas.

El segundo momento surgió cuando las condiciones políticas del Ecuador comenzaron a girar con la llegada de Lenín Moreno a la Presidencia de la República, quien, a pesar de pertenecer al mismo partido de Correa, acogió algunos pedidos de la prensa para mejorar las relaciones. Uno de ellos es la reforma a la Ley de Comunicación, publicada en el Registro Oficial en febrero de 2019, que eliminó varios artículos que eran cuestionados, como la figura del linchamiento mediático y la Superintendencia de Comunicación. Esta última se encargaba de sancionar a los medios de comunicación y a los periodistas con dinero, rectificaciones o correcciones constantes. Otra decisión tomada, pero por la Corte Constitucional en 2018, fue derogar la declaratoria de la comunicación como servicio público, junto con otro grupo de reformas constitucionales hechas en 2015.

Paralelamente, el Gobierno impulsó una reforma política que llevó a que se reemplazaran a varias autoridades del Estado, especialmente de los organismos de control del Estado (Fiscalía, Contraloría y superintendencias), de la justicia y de la defensa de derechos humanos. 
Esta imagen del gobierno de Moreno, de reconstrucción del sistema democrático, también hizo que las ONG internacionales vieran que las condiciones cambiaban en el país, por lo que su apoyo también dio un nuevo giro. Al momento, los temas ambientales, de mujer y migración son los que más tienen cabida en la agenda internacional de la cooperación y los cibermedios lo saben, por lo que presentan propuestas (en proyectos concursables) vinculadas al trabajo de alguna ONG que cuente con fondos para que financie algún trabajo periodístico.

\subsection{Los casos de Plan V y Periodismo de Investigación}

Plan V comenzó en 2013 como una página de Facebook y, en septiembre de ese año, ya tenían un portal. Esto fue posible por el apoyo de Fundamedios, que donó los equipos, mientras ellos se constituían como una cooperativa de periodistas, aunque esto último no prosperó.

Durante su búsqueda de financiamiento presentaron algunos proyectos que fueron remitidos a Open Society, Universidad de Texas, Centro Carter, Pulitzer Foundation, NED, Fundamedios, además de los organismos de cooperación de España, Noruega y Holanda.

La NED, que es una iniciativa del Congreso de los Estados Unidos para promover la democracia liberal, contestó y, según Juan Carlos Calderón, director de Plan V, esto se debió a que su propuesta claramente planteaba que le interesaba recuperar la democracia. A partir de ese apoyo, prepararon nuevos proyectos dirigidos a más organismos que les pudiera interesar el trabajo de Plan V. Esto ha hecho que este cibermedio se conciba a sí mismo como una empresa de venta de servicios comunicacionales a empresas grandes, lo cual les garantiza su supervivencia y el pago de sus deudas. Su línea editorial se ha visto condicionada a los fondos que puedan encontrarse.

En palabras de Calderón, mantienen el principio de reportear, de estar en el lugar de los hechos, de trabajar con fuentes oficiales y no oficiales, pero en la agenda temática sí hay temas que responden a los proyectos por los cuales reciben fondos.

Para superar el conflicto ético y de ejercicio periodístico que pudiera significar esto, el portal ha optado por plantear un tema amplio mientras buscan qué institución puede interesarse en esos contenidos, como ocurrió con una investigación sobre violaciones de derechos humanos, que interesó a la OEA.

Esto tampoco ha hecho que no acojan los pedidos de investigaciones concretas, como las vinculadas al narcotráfico, en las que ellos definen cómo abordar el tema y cómo presentarlo. Si se hace relación con los modelos de negocios antes presentados, esta forma de trabajo refuerza la idea de que este 
cibermedio ha optado por un sistema patrocinado.

Periodismo de Investigación comparte con Plan V el apoyo recibido por la NED. Este cibermedio surge con el apoyo de Mil Hojas y La Fuente, dos portales que funden sus esfuerzos investigativos, y que recibieron, en un principio, apoyos del sector privado que no han sido identificados, por pedido de los propios aportantes. Una de las razones de esto es la presión ejercida durante el gobierno de Rafael Correa, a través del Sistema de Rentas Internas, el Ministerio de Trabajo o en el Instituto de Seguridad Social, en contra de quienes daban apoyo a los periodistas.

En el caso de Periodismo de Investigación, su fortaleza para conseguir fondos de las ONG está en sus contenidos, que tienen la capacidad de marcar la agenda política nacional e impulsar el debate de la opinión pública del país, en medios masivos tradicionales y en las redes sociales. Esto permite que se muestren como clave en el activismo social, lo que también es otro punto de atracción para las ONG.

Su práctica periodística está concentrada en la investigación de la corrupción de cuello blanco, aunque no siempre logran la contrastación de la información, por el riesgo que implica hacerlo y la posibilidad de que los funcionarios o exfuncionarios del Gobierno, con sus prórrogas, congelen los temas, según explica Zurita.

Tabla 1. Comparaciones de los dos medios analizados

\begin{tabular}{|c|c|c|}
\hline & Plan V & $\begin{array}{l}\text { Periodismo de } \\
\text { investigacion }\end{array}$ \\
\hline $\begin{array}{l}\text { Periodicidad de } \\
\text { publicación }\end{array}$ & $\begin{array}{l}\text { Semanal, pero actualiza } \\
\text { información relevante }\end{array}$ & $\begin{array}{l}\text { Una vez finalizada la } \\
\text { Investigación }\end{array}$ \\
\hline Temática & Generalista & Corrupción \\
\hline Secciones & $\begin{array}{l}\text { Investigación, historias, } \\
\text { ideas, política, entrevista, } \\
\text { sociedad, confidenciales }\end{array}$ & Casos e historias \\
\hline Uso de redes sociales & $\begin{array}{c}\text { Facebook, Twitter, } \\
\text { Instagram y Whatsapp }\end{array}$ & $\begin{array}{c}\text { Facebook, Twitter e } \\
\text { Instagram }\end{array}$ \\
\hline Multimedios & Texto, vídeo y fotografía & Texto, vídeo y fotografía \\
\hline
\end{tabular}

Fuente y elaboración propias. 


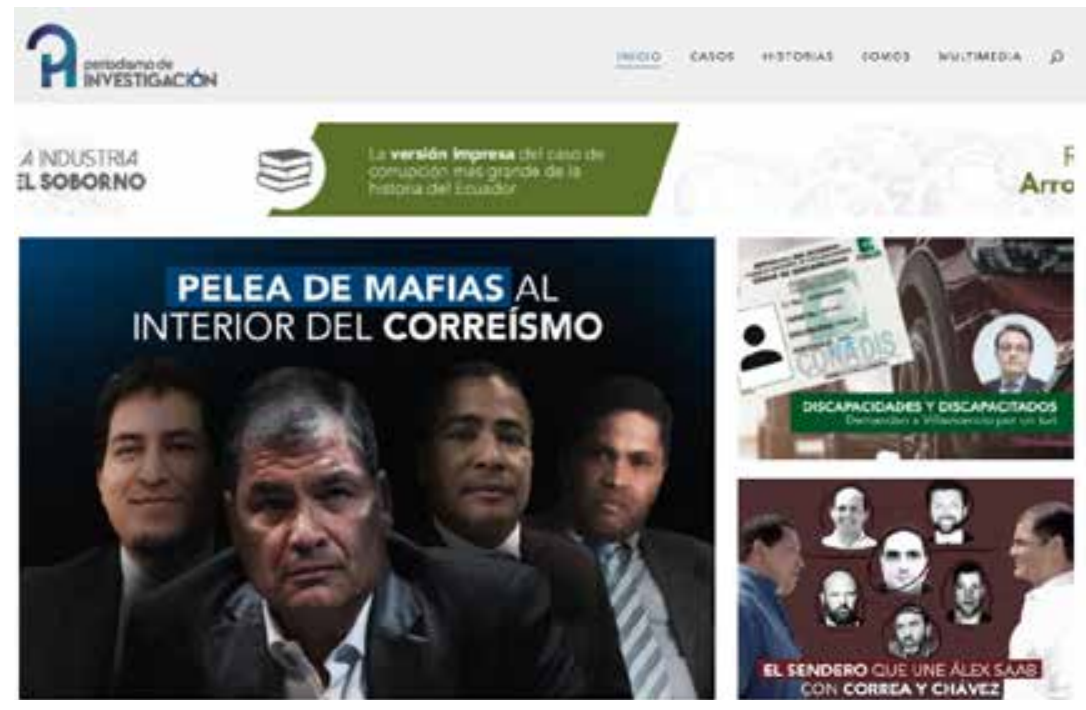

Periodismo de Investigación ha centrado su trabajo en denuncias de corrupción y, desde su aparecimiento, se ha centrado en el gobierno del expresidente Rafael Correa. En más de una ocasión han admitido que tienen apoyo de las ONG.

En la Tabla 1, si se parte de un análisis de los puntos en común, se muestra que los dos cibermedios optan por un uso parcial de multimedios y ambos utilizan los mismos: textos, vídeo y fotografía. Normalmente, los reportajes que se mantienen en la página de inicio son los más polémicos y, en el caso de Periodismo de Investigación, no hay actualización de noticias, es decir prioriza exclusivamente sus reportajes o informes. Plan V trata de mantener información actualizada, a través de crónicas o entrevistas. Sus actualizaciones más importantes se dan dos días a la semana: el jueves para los columnistas de opinión y el viernes para la información producida por ellos, por lo menos así lo reciben quienes mantienen suscripción al servicio de actualización del portal.

En ese mismo espacio informativo, es decir Plan V, es notoria la presencia de información relativa a los derechos humanos, expresados en las secciones de Historias y Sociedad. Periodismo de Investigación es más monotemático: se concentra en las denuncias alrededor de hechos de corrupción y no les preocupa tener una actualización de la información diaria.

El uso de redes sociales es más amplio en Plan V, ya que utiliza cuatro canales; sin embargo, Periodismo de Investigación tiene un gran aliado en Twitter, pues sus dos principales responsables, Fernando Villavicencio y Christian Zurita, están constantemente en ese espacio generando debate. Normalmente 
hay viralización de sus contenidos y su número de seguidores en Twitter es de 41,6 mil para el primero y 36,6 mil para el segundo, de acuerdo con la medición hecha en noviembre de 2019.

\section{Conclusiones}

Los modelos de negocios digitales que plantean Campos Freire y Pavlik (2015), y que recogen la postura de Picard (2011), se aplican, en general, a la realidad ecuatoriana. Sin embargo, dentro del esquema de patrocinio, hay que destacar la presencia de los fondos internacionales, provenientes de la cooperación conseguida en las ONG internacionales, a través del apoyo, primero de las ONG locales, y actualmente en relación directa con esos organismos por parte de los cibermedios.

La presencia de apoyo internacional en el ciberespacio mediático ecuatoriano se produjo en una coyuntura específica: un gobierno autoritario que había establecido una relación compleja y tensa con los periodistas y los medios de comunicación tradicional. Los periodistas y su trabajo fueron fuertemente cuestionados e, incluso, acusados de falta de profesionalismo, de ser tendenciosos y opositores al gobierno de turno. La normativa aprobada alrededor de la comunicación, y específicamente de la prensa, fue para sancionar económicamente a los medios y periodistas, lo que llevó a una falta de garantías para el ejercicio profesional y, consecuentemente, que se instale la autocensura.

En medio de este escenario, los periodistas salieron de las salas de redacción y vieron en el espacio web una forma de seguir con su trabajo. Pero sabían que iban a necesitar ayuda, especialmente para que su actividad les genere ingresos tanto para vivir como para mantener al aire sus producciones.

Si el escenario era difícil para los periodistas y los medios de comunicación informativos, también lo era para los dirigentes sociales y las organizaciones no gubernamentales. Estas fueron sometidas a escrutinio y, sobre todo, vigiladas en sus actividades. Las que eran consideradas opositoras al gobierno fueron denunciadas y se llegó a disolver dos: Acción Ecológica y la Unión Nacional de Educadores. Fundamedios fue amenazada con su eliminación.

Esta última ONG fue clave en los primeros años en el surgimiento de los cibermedios: denunció la situación de la prensa ante las Relatorías de la Libertad de Expresión de la ONU y la OEA. Esto llamó la atención de las ONG internacionales que pusieron en su radar al Ecuador, brindaron capacitación y ubicaron los fondos semillas para cuatro medios. Luego, se sumó el FOPE, otra ONG local, al trabajo de capacitación de periodistas ecuatorianos. 
Con el cambio de la coyuntura política en mayo de 2017 y la reforma al sistema político y de comunicación del país, las ONG internacionales quitaron de las prioridades proyectos y fondos vinculados a la defensa de la libertad de prensa y de expresión, así como el fortalecimiento del sistema democrático, y dieron paso al apoyo de otra temática vinculada también a derechos humanos como es medio ambiente, migración, mujeres, además, de lucha contra la corrupción.

Plan V nació en plena crisis entre el gobierno de Rafael Correa y la prensa, mientras que Periodismo de Investigación bastante tiempo después, en 2018. El primer medio admite que su agenda temática está influenciada por los fondos que puede conseguir. Al definirse como una empresa de comunicación, considera válido y viable hacer periodismo sobre la temática que tiene el interés de los cooperantes y así mantener su negocio. En el caso de Periodismo de Investigación, sus representantes no se sienten atados y afirman que responden únicamente a su criterio periodístico, pero admiten que tienen soportes de las ONG y su trabajo es monotemático: lucha contra la corrupción, que justamente es uno de los temas que interesa a la comunidad internacional.

En suma, los resultados de esta investigación permiten concluir que, en circunstancias como las que atravesó el Ecuador, la cooperación internacional es una alternativa viable para mantener al periodismo libre. Una vez que los gobiernos autoritarios y las circunstancias cambian, el mantener una agenda setting cercana a las líneas de apoyo de esos mismos organismos revela que sigue siendo necesario el apoyo de fundaciones para que el periodismo continúe con su trabajo, ante la falta de capacidad de gestión de estos cibermedios y de un modelo de negocio rentable. 


\section{Referencias}

Álvarez, A. y Chumaceiro, I. (2012). Insulto e intolerancia: La confrontación en el macro diálogo político. En D. L. Pessoa de Barros (Ed.), Preconceito e Intolerancia: reflexões lingüístico discursivas (pp. 137-176). Sao Paulo: Editora Mackenzie.

Amado, A. (2015). Calidad periodística y fuentes presidenciales: el periodismo argentino frente a la comunicación de gobierno. Estudios sobre el Mensaje Periodístico, 21, 63-84. doi: 10.5209/rev_ESMP.2015.v21.50656

Ayllón, B. y Dolcetti, M. (2014). Revolución Ciudadana, Buen Vivir y Cooperación en Ecuador (2007-2013). Relaciones Internacionales, 23(46), 177-199. Recuperado de https://revistas.unlp.edu.ar/RRII-IRI/article/view/779

Campos Freire, F. (2015). Journalism and Metamedia. En X. L. García y F. Campos Freire (Eds.), Journalism in Change (pp. 91-121). Porto: Media XXI.

De la Torre, C. (2015). De Velasco a Correa. Insurrecciones, populismos y elecciones en Ecuador, 1944-2013. Quito: Corporación Editora Nacional y Universidad Andina Simón Bolívar.

Escandón-Montenegro, P. y Levoyer-Salas, S. (2019). Ecuador: Ciberperiodismo de investigación y agenda de la cooperación internacional. ComHumanitas: Revista Científica De Comunicación, 10 (2), 8-29. doi: 10.31207/rch. v10i2.196

Levoyer, S. (2019). El 30 de septiembre de 2010: un análisis de las visiones y de los discursos de los diarios Hoy y El Telégrafo (Tesis doctoral, Universidad Santiago de Compostela). Recuperado de http://hdl.handle. net/10347/19864

López, X. y Pereira, X. (2008). Alternativas en los medios de comunicación digitales. Televisión, radio, prensa, revistas culturales y calidad de la democracia. Barcelona: Gedisa.

Mendieta Vicuña, D. y Esparcia Pérez, J. (2018). Aproximación metodológica al análisis de contenido, a partir del discurso de los actores. Un ensayo de investigación social de procesos de desarrollo local (Loja, Ecuador). Revista Empiria. Revista de Metodología de Ciencias Sociales, (39), 15-47. doi: 10.5944/empiria.39.2018.20876

Pavlik, J. (2008). Media in the Digital Age. New York: Columbia University Press. 
Picard, R. G. (2011). Mapping digital media: Digitization and media business models. London: Open Society Foundations. Recuperado de https:// www.opensocietyfoundations.org/reports/digitization-media-business-models

Portilla Manjón, I., Vara-Miguel, A. y Díaz-Espina, C. (2016). Innovación, modelos de negocio y medición de audiencias ante los nuevos retos del mercado de la comunicación. En C. Sábada, J. A. García y M. P. Martínez-Costa (Coords.), Innovación y desarrollo de los cibermedios en España (pp. 31-39). Pamplona: Eunsa.

Punín, M. I., Martínez, A. y Rencoret, N. (2013). Medios digitales en Ecuador: perspectivas de futuro. Revista Comunicar, XXI (42), 199-207. doi: 10.3916/C42-2014-2

Rincón, O. (2010). ¿Hay que defender a los medios de comunicación del Estado o al Estado de los medios y de los periodistas? En O. Rincón (Ed.), ¿Por qué nos odian tanto? (Estados y medios de comunicación en América Latina) (pp. 5-13). Bogotá: Centro de Competencia en Comunicación para América Latina y Friederich Ebert Stiftung.

Rodríguez Gelfenstein, S. (2013, 5 de octubre). Ecuador: una política exterior soberana que resiste los embates del imperio. El Telégrafo. Recuperado de https://www.eltelegrafo.com.ec/noticias/columnistas/15/ecuador-una-politica-exterior-soberana-que-resiste-los-embates-del-imperio

Sábada, C. (2016). Modelos de negocio y coordinación multiplataforma. En C. Sábada, J. A. García y M. P. Martínez-Costa (Coords.), Innovación y desarrollo de los cibermedios en España (pp. 247-254). Pamplona: EUNSA.

Salaverría, R. (2005). Redacción periodística en Internet. Pamplona: EUNSA.

Senplades. (2010). Plan Nacional de Desarrollo (2007-2010). Quito: Senplades. 